\title{
ULTRASONOGRAFIA DE APOYO EN TECNICAS DIAGNOSTICAS Y TERAPEUTICAS
}

\author{
Dr. Patricio Palavecino R.
}

Unidad de Radiología Intervencional. Centro de Imagenología. Hospital Clínico, Universidad de Chile.

\begin{abstract}
Diagnostic and palliative procedures performed by Interventional Radiologists and guided by ultrasound are analyzed. An important issue is the clinical evaluation of the patient previous to the procedure, standardized protocols and post procedure follow-up.
\end{abstract}

Key words: Interventional radiology, Ultrasound.

Resumen: Los procedimientos diagnósticos y paliativos guiados por ultrasonografía son analizados; entre ellos biopsias, ablación de tumores, drenajes y accesos venosos. Especial énfasis se da a la evaluación del paciente previo y posterior al procedimiento, de igual forma a la existencia de protocolos estandarizados.

Palabras claves: Radiología intervencional, Ultrasonido.

\section{Introducción}

El desarrollo tecnológico ha beneficiado en forma importante al diagnóstico por imágenes permitiendo identificar lesiones con extraordinaria precisión espacial, ello ha permitido un gran avance de las técnicas intervencionistas, evitando en algunos casos cirugías mayores, disminuyendo la morbimortalidad, días de hospitalización y costo total de un tratamiento.

Actualmente en muchas unidades de intervencionismo de los servicios de imagenología, se realizan procedimientos guiados por ecografía, tomografía computada (TC), fluoroscopia y resonancia magnética. En nuestra unidad se realizan una serie de técnicas diagnósticas y terapéuticas guiadas por ultrasonido (US) tales como: biopsias, drenaje de colecciones, nefrostomías y tratamientos percutáneos de tumores con ablación por radiofrecuencia y alcoholización entre las más frecuentes. Para este objetivo, se ha protocolizado

Palavecino $\boldsymbol{P}$. Ultrasonografia de apoyo en técnicas diagnósticas y terapéuticas. Rev Chil Radiol 2004; 10 : 132-138.

Correspondencia: Dr. Patricio Palavecino R. Santos Dumont 999

E-mail:ppalavecino@ns.hospital.uchile.cl el manejo del paciente, previo, durante y después del procedimiento, estableciendo normas claras que se deben cumplir para garantizar su éxito.

\section{Evaluación previa al procedimiento}

Es responsabilidad del radiólogo que realizará la técnica intervencional, conocer previamente al paciente, su historia clínica, solicitar la firma del consentimiento informado y contactar a sus familiares si fuese necesario.

La evaluación de las condiciones generales del paciente a consignar comprenden: Conocimiento de su evolución clínica, su estado de conciencia, y antecedentes mórbidos de importancia tales como diabetes mellitus, hipertensión arterial, mieloma, cardiopatías, insuficiencia renal, hepática, o coagulopatías. De igual forma hay que tomar conocimiento de los exámenes radiológicos y de laboratorio.

Los exámenes de laboratorio incluyen: Hemograma con recuento de plaquetas, actividad de protrombina, tiempo de protrombina, tiempo parcial de tromboplastina y electrocardiograma en pacientes mayores de 50 años.

En casos de tratamiento con heparina debe suspenderse 4 horas antes, y en los con dicumarínicos 2 días antes repitiendo previo al procedimiento las pruebas de coagulación. Tratamientos con fibrinolíticos contraindican todos los procedimientos invasivos. El uso de aspirina se debe suspender 7 días antes del procedimiento.

Es necesario un ayuno previo de a lo menos 6 horas, en aquellos pacientes que requieren analgesia, sedación o apoyo anestésico.

La mayoría de los procedimientos, a excepción de aquellos menos complejos, hacen obligatorio tener una vía venosa permeable y monitorización con control electrocardiográfico, oximetría, presión arterial y pulso durante el proceso. En pacientes en que se utilice contraste yodado endovenoso, investigar: Creatinemia reacciones adversas, alergias conocidas, asma. En algunos procedimientos tales como; drenajes biliares, nefrostomías, derivación portosistémico transyugular (TIPS) entre otros; es necesario el uso de antibióticos profilácticos antes y después de él. 


\section{Consideraciones posteriores al procedimiento}

La responsabilidad del equipo radiológico intervencional no termina con el procedimiento, es necesario un estrecho seguimiento del paciente.

1. Se debe evaluar el estado y algunos parámetros tales como signos vitales y el estado general con relación a las condiciones previas para observar su evolución. Las complicaciones durante el procedimiento, deben informarse al médico tratante y consignarse en la historia clínica y en la ficha de radiología intervencional.

2. Se atenderá al paciente médicamente hasta que salga de la sala de Radiología Intervencional efectuando compresión en el sitio de punción, fijación de drenajes, etc. Se anotará además en la ficha clínica la descripción del procedimiento realizado, sus resultados, indicaciones y tratamiento a seguir.

\section{A. Estudio y tratamiento de patología tumoral \\ A.1. Biopsias guiadas por US}

Para el control de biopsias, la US posee una serie de conocidas ventajas como son; bajo costo, portabilidad, disponibilidad en la mayoría de los hospitales, guía en todos los planos del espacio y entrega de información en tiempo real permitiendo una buena visualización de la aguja. La mayoría de los equipos poseen la opción Doppler color que informa respecto de la vascularización de la lesión. Bien sabido es el hecho que la US es dependiente del operador.

Las biopsias percutáneas, con aguja fina 20 a $25 \mathrm{G}$, para obtener muestras para estudio citológico o con algunas de mayor calibre 14 a $19 \mathrm{G}$, para análisis histológico; gozan de gran aceptación por todos los médicos, principalmente por ser una técnica precisa, segura y en la mayoría de los casos capaz de caracterizar la enfermedad primaria o metastásica. Los diversos estudios han demostrado que el diagnóstico histológico se puede realizar en el $90 \%$ de los casos incluso en tumores menores a $1.5 \mathrm{~cm}^{(1)}$.

Las biopsias generalmente están indicadas para confirmar el diagnóstico de una masa o nódulo de cualquier órgano de la economía que sea factible de puncionar. Hay casos que una masa impresiona como benigna, pero será finalmente el procedimiento biópsico, el que definirá su etiología. En general la biopsia con aguja no tiene contraindicaciones absolutas, existen algunas relativas, susceptibles de manejar; como lo son: falta de colaboración del paciente, coagulopatías o ausencia de un trayecto adecuado, ya sea por interposición de asas intestinales o presencia de grandes vasos.

La punción biopsia con aguja guiada por US se emplea en muchos órganos y es óptima en aquellos superficiales. Nosotros la hemos utilizado en tiroides (Figura 1), paratiroides, riñón, glándulas suprarrenales, masas para-vertebrales y de partes blandas, páncreas, hígado (Figura 2), y otros órganos abdominales y pelvianos en que la lesión pueda ser adecuadamente visualizada.

La especificidad del procedimiento oscila entre 70 a $98 \%$ según diversas publicaciones dependiendo de la localización, tamaño y tipo histológico entre otros factores ${ }^{(1,2)}$. Existen pocas complicaciones con tasas de mortalidad menores a $0.031 \%^{(3)}$. De las complicaciones graves, las hemorragias aunque
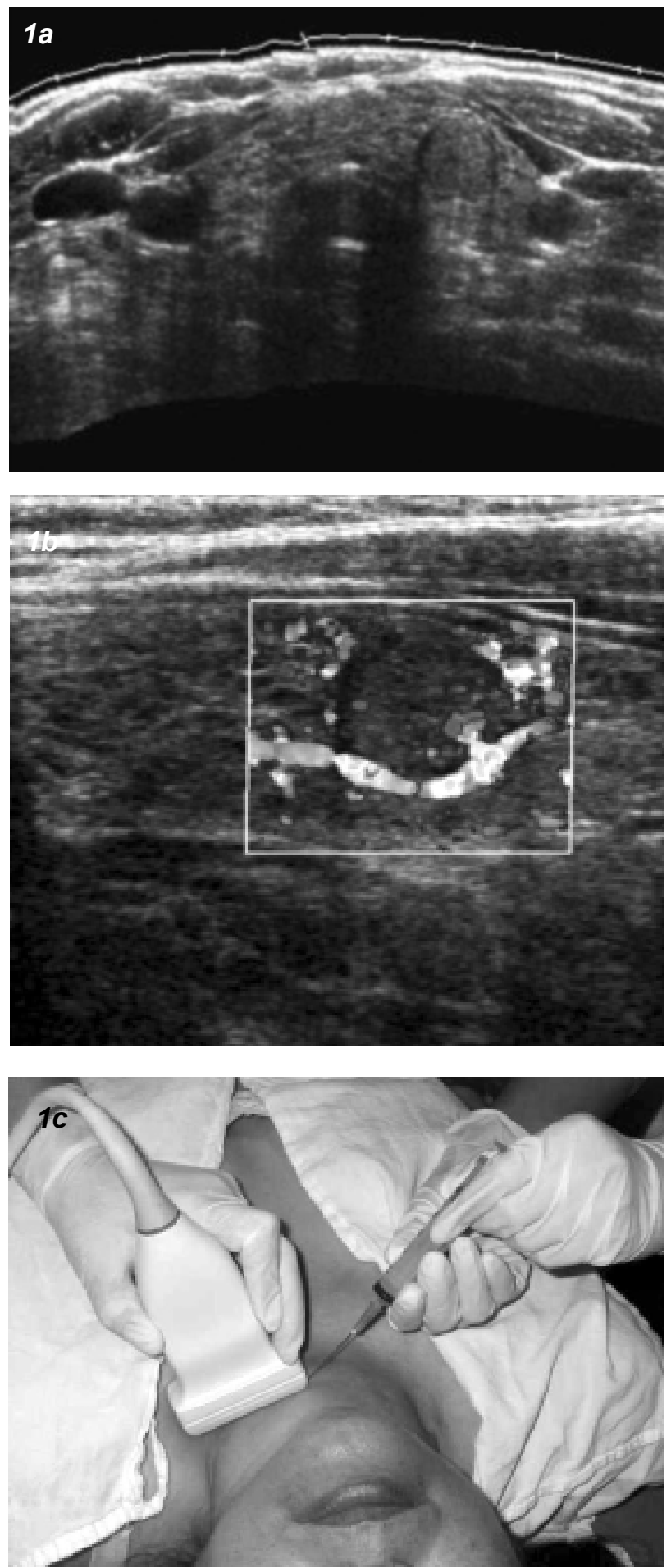

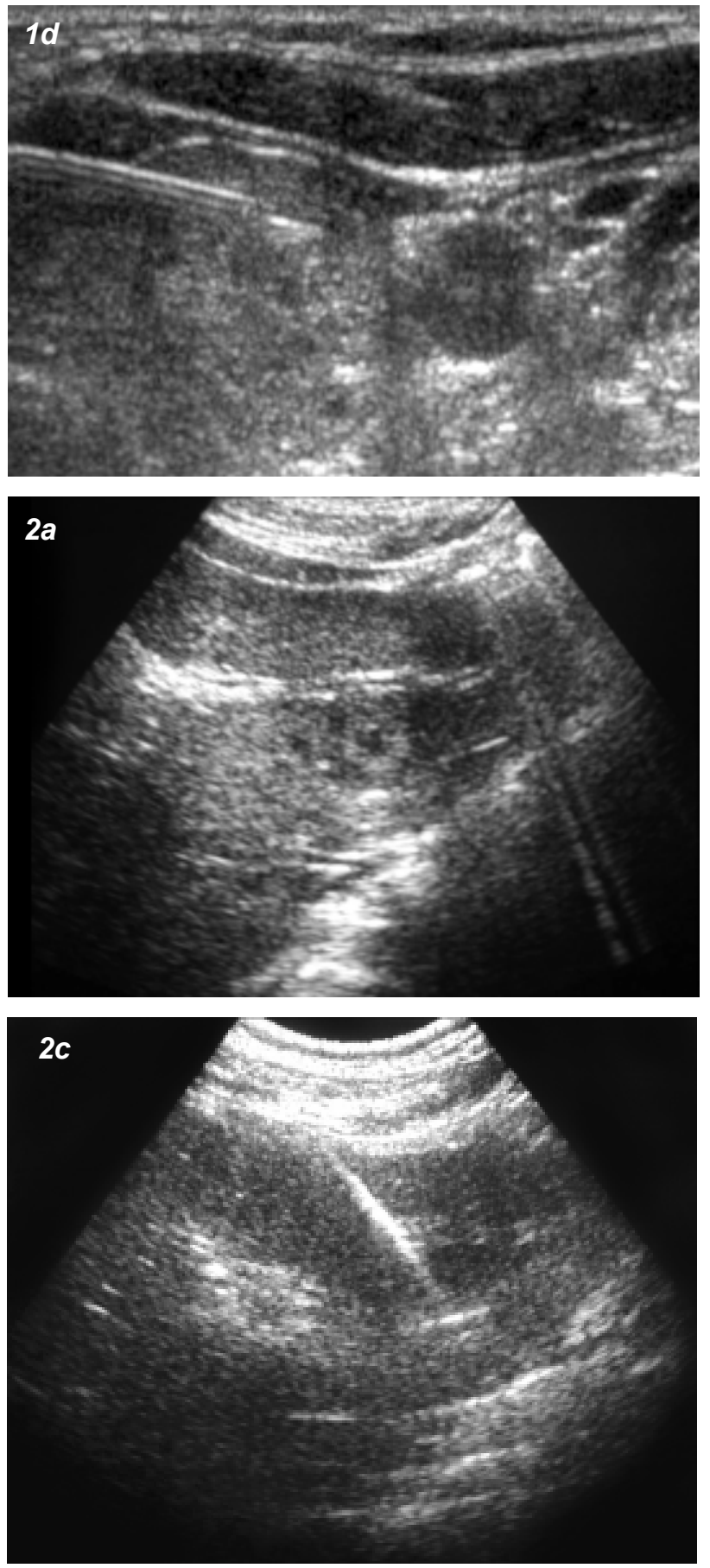

raras se ven asociadas a biopsias de órganos sólidos, otras complicaciones secundarias descritas son neumotórax, pancreatitis, peritonitis, hemobilia y en algunos casos diseminación del tumor a través del trayecto con una incidencia reportada de 0.005 a $3.4 \%{ }^{(3,4)}$.

\section{A. 2. Ablación de tumores}

Las posibilidades de tratamiento que actualmente tienen los pacientes con tumores primarios hepáticos, renales o con metástasis de tumores de colon o mama se han incrementado debido a los avances tanto en la cirugía, como en el
Figura 1 a-d. Punción tiroidea guiada por ecografía. a: US corte transversal demostrando un nódulo sólido en lóbulo izquierdo. b: US Doppler color mostrando la vascularización del nódulo. c,d: Punción con aguja fina. Técnica (c) y guía ultrasonográfica demostrando la aguja como una imagen lineal ecogénica (d).

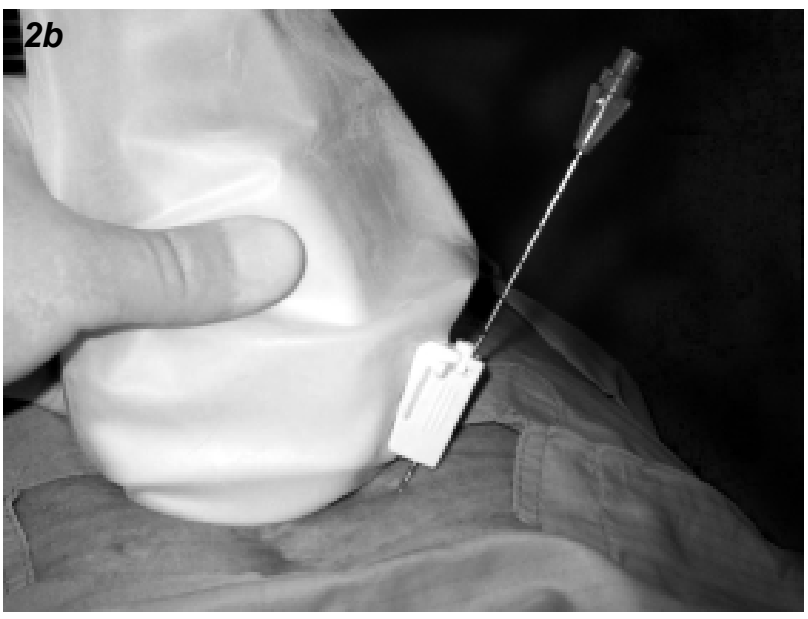

Figura 2 a-c. Punción de nódulo hepático. a: US demostrando formación nodular sólida hepática b: Punción con aguja gruesa. c: Visión ecográfica de la aguja como imagen lineal ecogénica en el espesor de la lesión.

tratamiento oncológico y técnicas percutáneas mínimamente invasivas.

La resección quirúrgica sigue siendo la primera opción terapéutica, pero no es aplicable a todos los pacientes, principalmente aquellos con importante riesgo vital, ya sea por edad, enfermedades asociadas o coagulopatías.

Se han desarrollado en los últimos años diversos procedimientos de tratamientos percutáneos que tienen como objetivo la destrucción del tumor y mejorar la sobrevida de los pacientes ${ }^{(5)}$. Se incluyen en estas técnicas la alcoholización ${ }^{(6)}$ (Figura 3), administración de ácido acético intratumoral o de suero caliente, crioterapia, termo-destrucción intersticial con electrodos de microondas o fibras láser. Se ha descrito recientemente que la técnica de ablación con radiofrecuencia de tumores hepáticos ha tenido buenos resultados y aceptación como curativa en aquellos pacientes en quienes la resección quirúrgica esta contraindicada ${ }^{(7-11)}$. En radiofrecuencia se utiliza generadores de 50-200 W, electrodos de 14-17 G, con agujas simples ("cool tip"), "paraguas" u otras; habiendo de varias longitudes (Figura 4). El abordaje percutáneo puede ser guiado por US, TC o resonancia magnética.

Se indica en pacientes considerados de alto riesgo quirúrgico, que tengan no más de cuatro lesiones menores a cinco centímetros y sin 

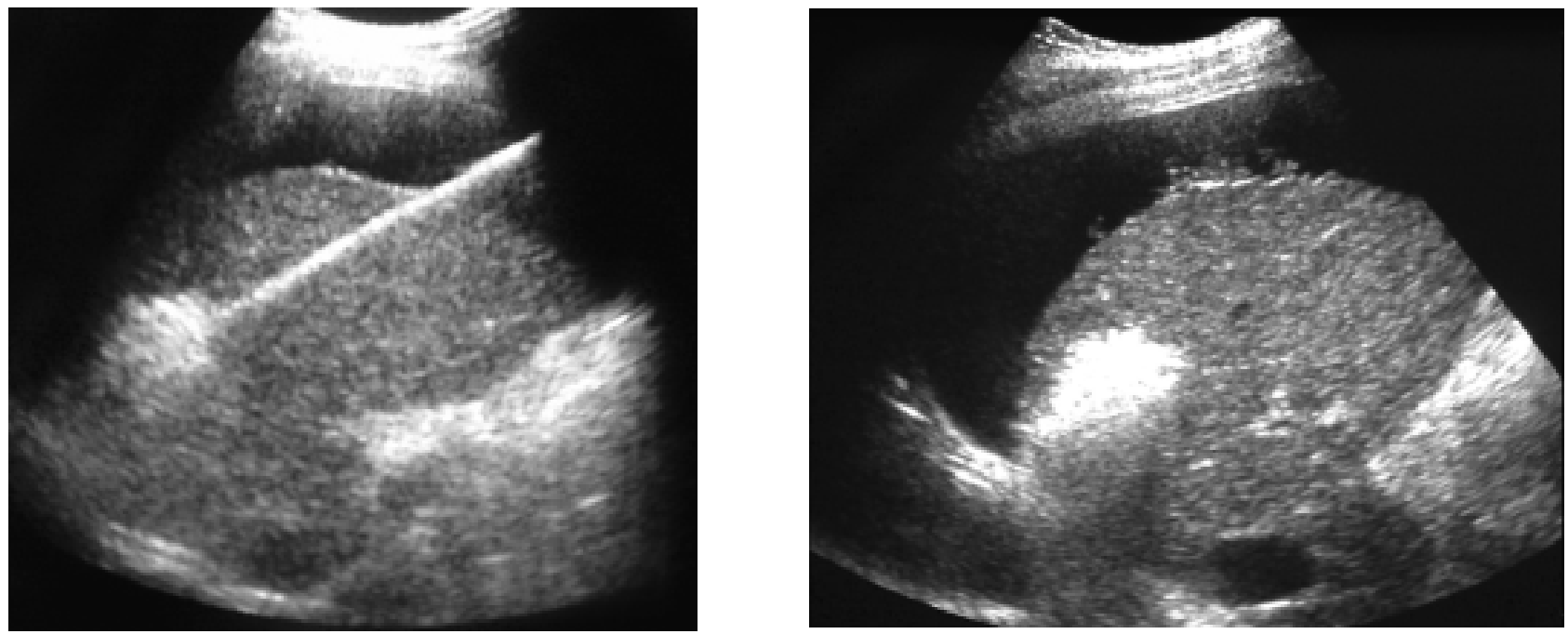

Figura 3 a,b. Alcoholización de tumor hepático guiada por ecografía. Paciente con ascitis.

enfermedad extrahepática. Está contraindicado en aquellos con enfermedades debilitantes, coagulopatías, lesiones próximas al hilio y aquellas no accesibles a través de la vía percutánea, prefiriendo en este caso el acceso por laparoscopia - laparotomía abierta (Figura 4 d). Se realiza seguimiento con imágenes a los siete días, 1, 3 y 6 meses.

Estos procedimientos tienen una morbilidad de alrededor de $0.8-2 \%$ con complicaciones tales como dolor, fiebre, hemorragias. En general no se describe mortalidad en las series estudiadas ${ }^{(8-11)}$.

\section{B. Drenaje percutáneo de colección guiada por US}

Se indica cada vez más dada su seguridad, eficacia y baja tasa de complicaciones, además se han desarrollado una serie de materiales que facilitan la técnica de aspiración diagnóstica o drenaje terapéutico de una colección ${ }^{(12)}$.

Con US es posible guiar el drenaje de colecciones de diversos órganos o cavidades .El éxito del procedimiento dependerá de: La localización de la colección que puede estar en un órgano o cavidad, las características morfológicas de ella que puede ser uni o multilocular, que este bien o mal delimitada y que sea profunda o superficial. En otros casos se puede estudiar la lesión con US y utilizar para guiar el drenaje; TC, fluoroscopia o resonancia magnética.

En la aspiración diagnóstica generalmente se utiliza agujas finas, tomando una pequeña cantidad de líquido que permitirá realizar estudios bioquímicos, citología y cultivos. En nuestra unidad ante la sospecha de infección, se efectúa estudio de Gram y se deja catéter de drenaje si fuera necesario.

La instalación de drenaje se realiza mediante la técnica de Seldinger o trocar, dependiendo de la experiencia del operador (Figura 5). La elección del catéter dependerá del tipo de órgano y consistencia de la colección. Es muy importante una adecuada evaluación clínica del paciente, elegir una vía de acceso muy segura incluyendo parénquima sano y se debe evitar órganos como el bazo, colon, intestino delgado y pleura. Existen contraindicaciones relativas como algunas coagulopatías, la sospecha de un quiste hidatídico por riesgo de anafilaxis o diseminación y la existencia de material no drenable como hematomas organizados ${ }^{(12,13)}$.

El manejo posterior requiere de un equipo de trabajo multidisciplinario actuando coordinadamente. La labor del radiólogo intervencionista comienza con la instalación del drenaje y termina cuando este es retirado; se evalúa periódicamente la posición del catéter manteniendo un drenaje adecuado de la colección, se debe desbridar y lavar cuando sea necesario y realizar fistulograma de la cavidad para su control.

Esta técnica se aplica a drenajes de abscesos hepáticos, pancreáticos o abdominopelvicos de diversas etiologías; pseudoquistes pancreáticos, drenajes transrectales, transvaginales o de la vía biliar, colecistostomías o nefrostomías (Figura 6) $)^{(14-}$ 22). En nuestra práctica diaria ya es habitual la punción de ascitis, derrames pleurales y otros (Figura 7).

\section{Acceso a vasos centrales con apoyo por US}

La indicación para la instalación de catéteres venosos centrales de larga permanencia incluye habitualmente la hemodiálisis, quimioterapia, antibióticoterapia y nutrición parenteral. Los abordajes más comunes son las venas yugular interna y la subclavia en casos excepcionales.

El índice de éxito de la punción de la vena yugular interna guiada por US (Figura 8) es cercana al $100 \%$ en la mayoría de los trabajos publicados, con pocas complicaciones generalmente pequeños 

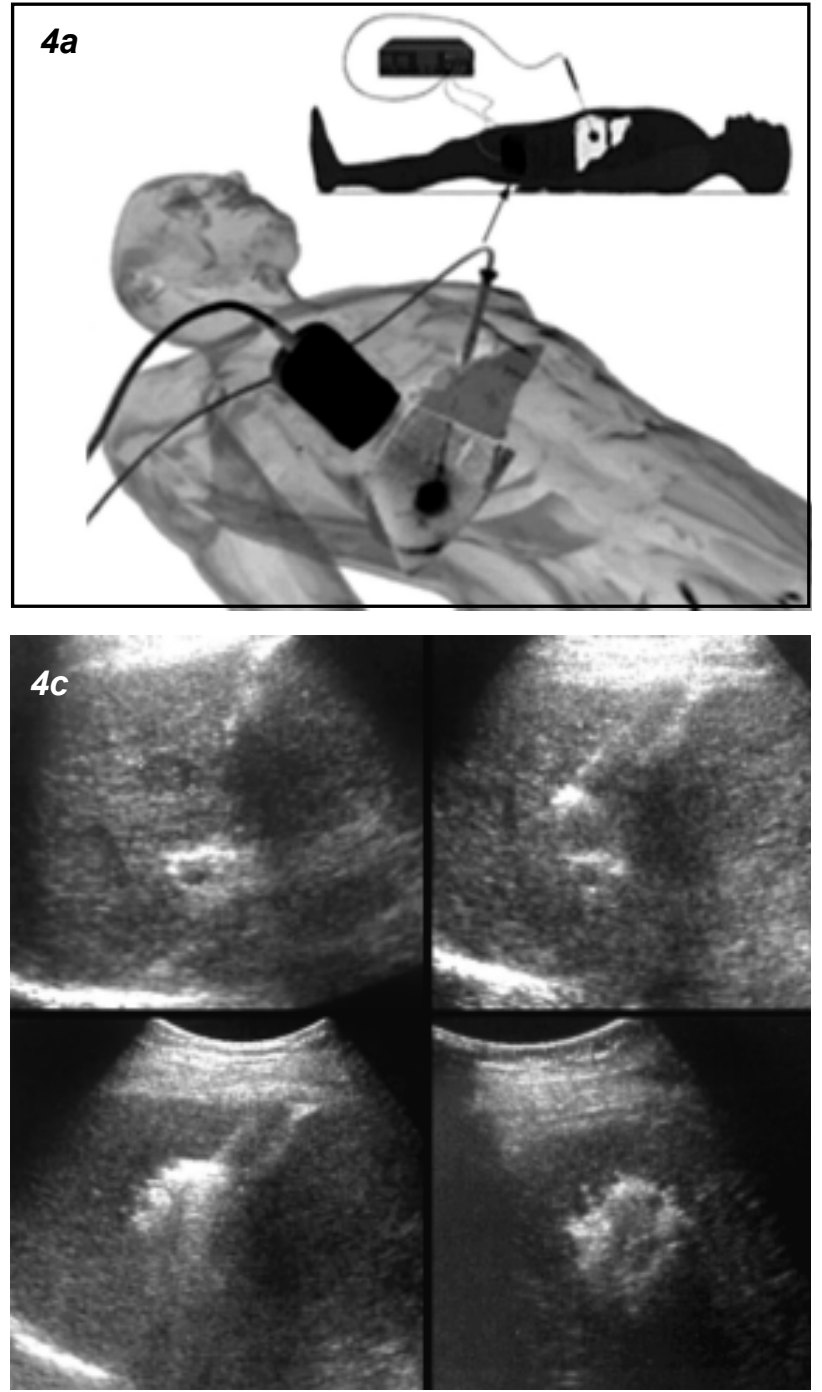

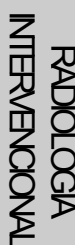

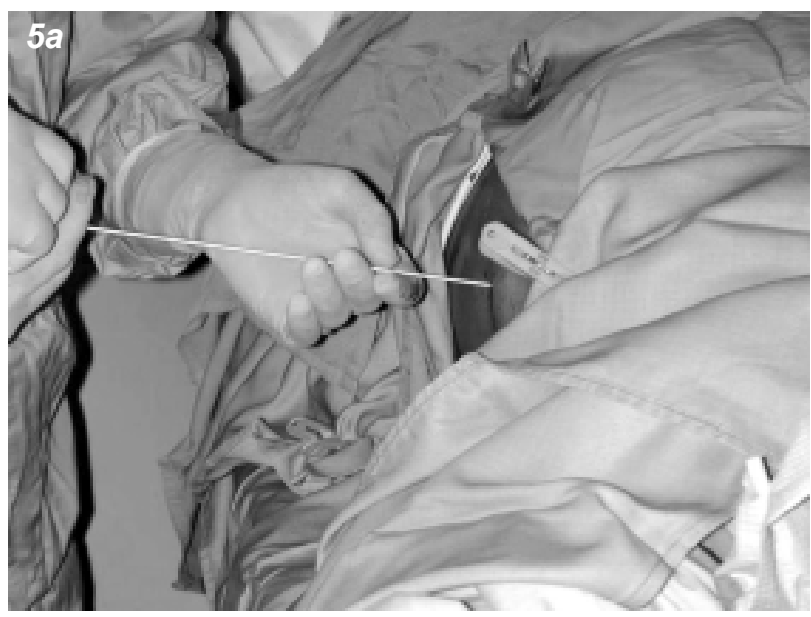

Figura 5 a-c. Técnica (a), esquema de drenaje (b) y control por TC de drenaje colección peripancreática (c).
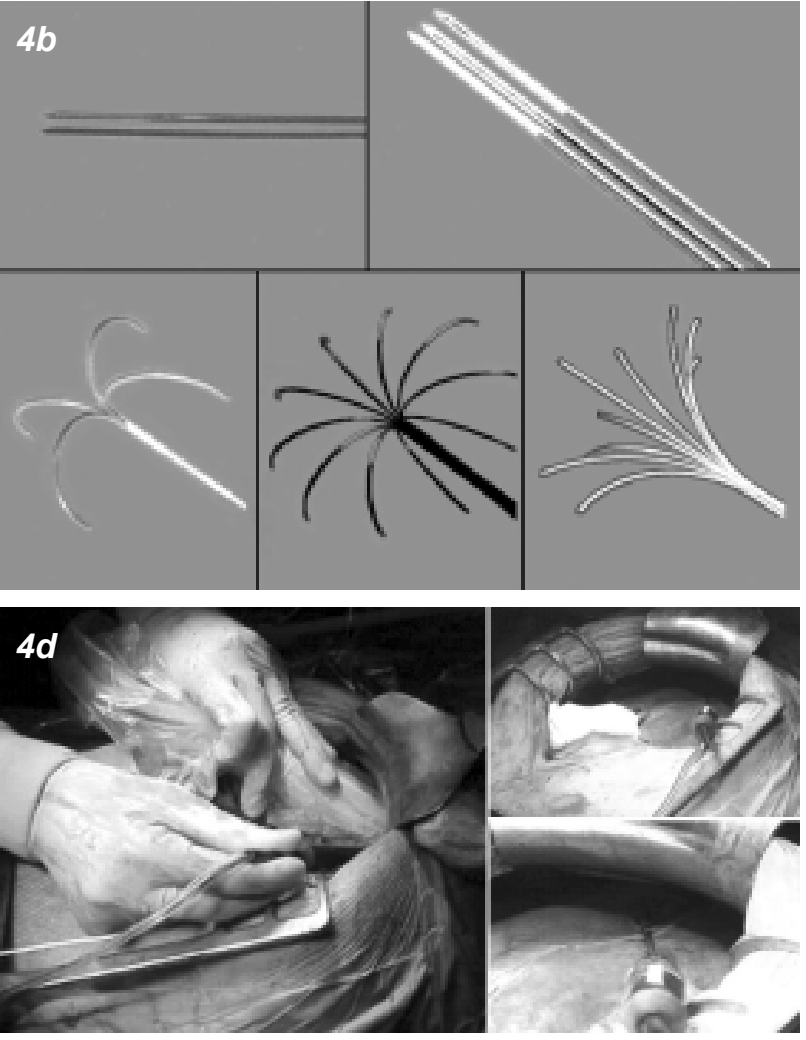

Figura 4 a-d. Ablación de tumor hepático con radiofrecuencia. a: Equipo y esquema del procedimiento de radiofrecuencia. b: Tipos de agujas utilizados en radiofrecuencia. c: Tu hepático en ablación por radiofrecuencia guiado por ecografía. d: Radiofrecuencia en paciente a través de laparotomía abierta.
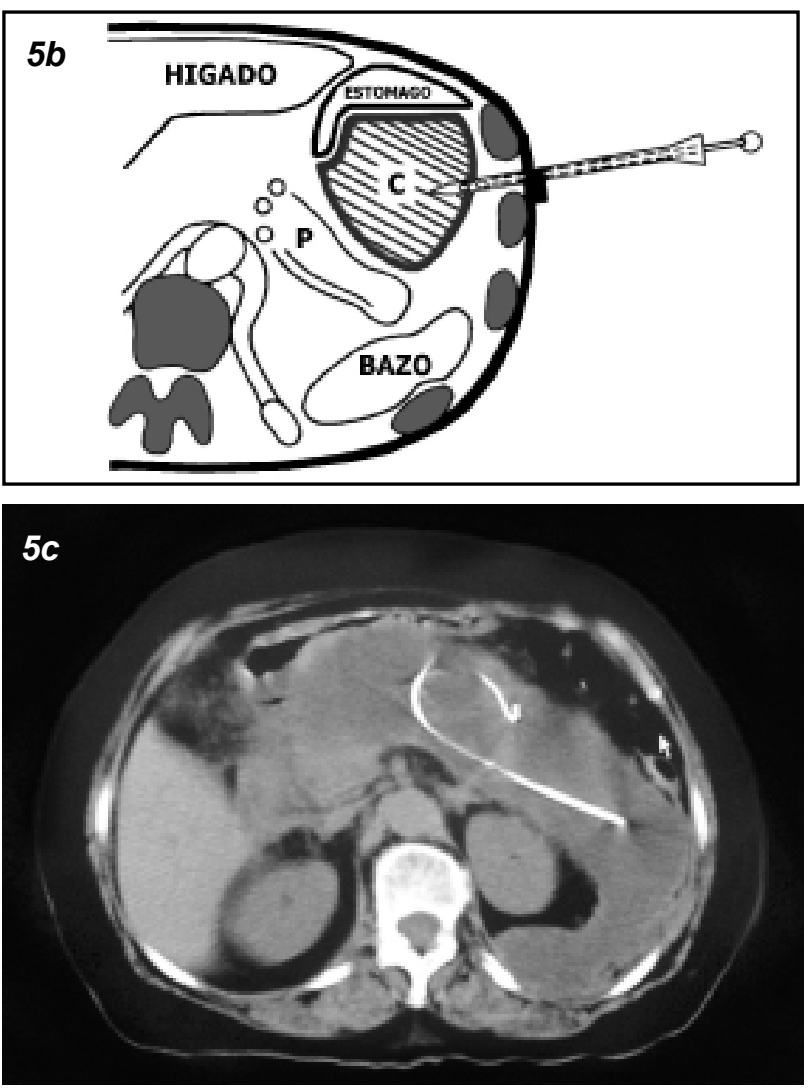

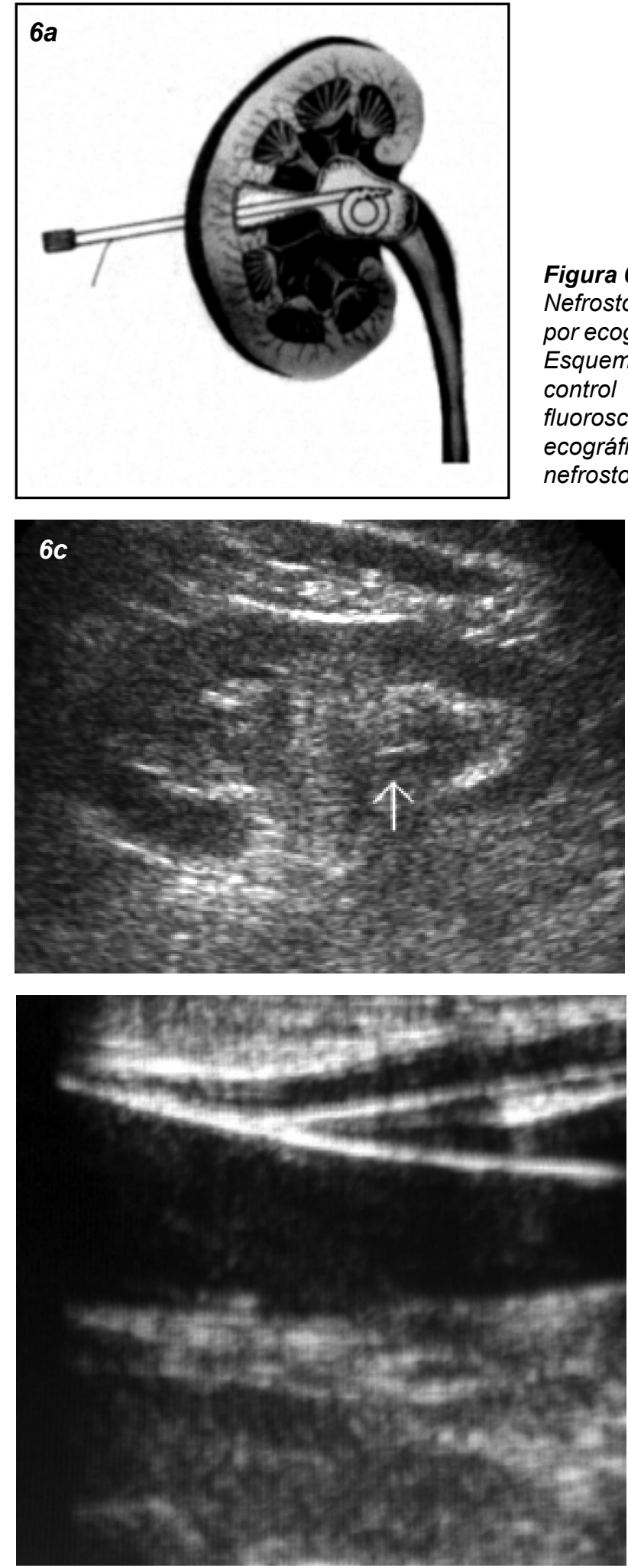

Figura 8. Control con US de acceso vascular a paciente que se realiza biopsia hepática transyugular.

hematomas y prácticamente ausencia de neumotórax ${ }^{(23)}$. Luego de puncionado el vaso el procedimiento se controla con apoyo fluoroscópico. También se puede acceder con este método a la vena femoral.
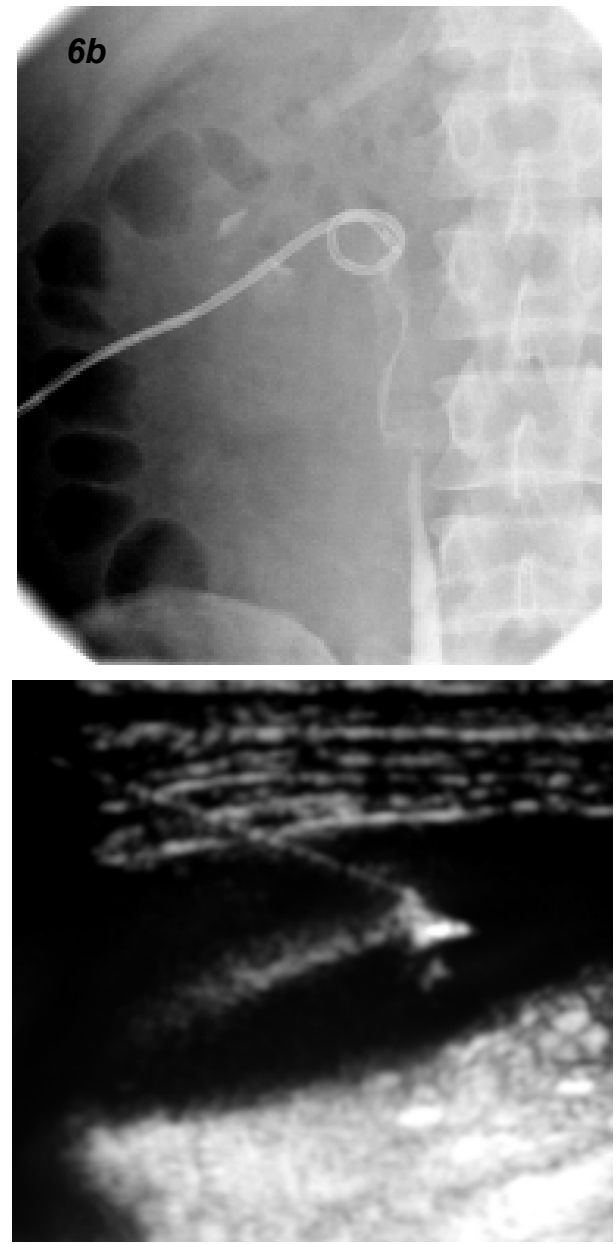

Figura 7. Punción de derrame pleural guiado por ultrasonido.

La técnica percutánea ha reducido los tiempos operatorios respecto a implantes quirúrgicos; además el vaso puede ser usado nuevamente, ya que no es ligado como sucede frecuentemente en la cirugía. EI US permite definir adecuadamente la anatomía de los vasos, variantes normales y presencia de trombos.

Algunos pacientes con insuficiencia renal crónica que requieren hemodiálisis, presentan trombosis múltiples de vasos periféricos e intentos fallidos de punción, siendo en ellos el apoyo con US fundamental. La instalación por métodos convencionales tiene un fracaso de alrededor de $10 \%$.

Hemos utilizado también este método de accesos venosos en pacientes que requieren de TIPS y/o punciones para obtener biopsias hepáticas por vía transyugular.

El ultrasonido permite también guiar el tratamiento de pseudoaneurismas arteriales postprocedimientos vasculares (Figura 9).

\section{Conclusión}

La US es un buen método para guiar procedimientos percutáneos tales como biopsias, 

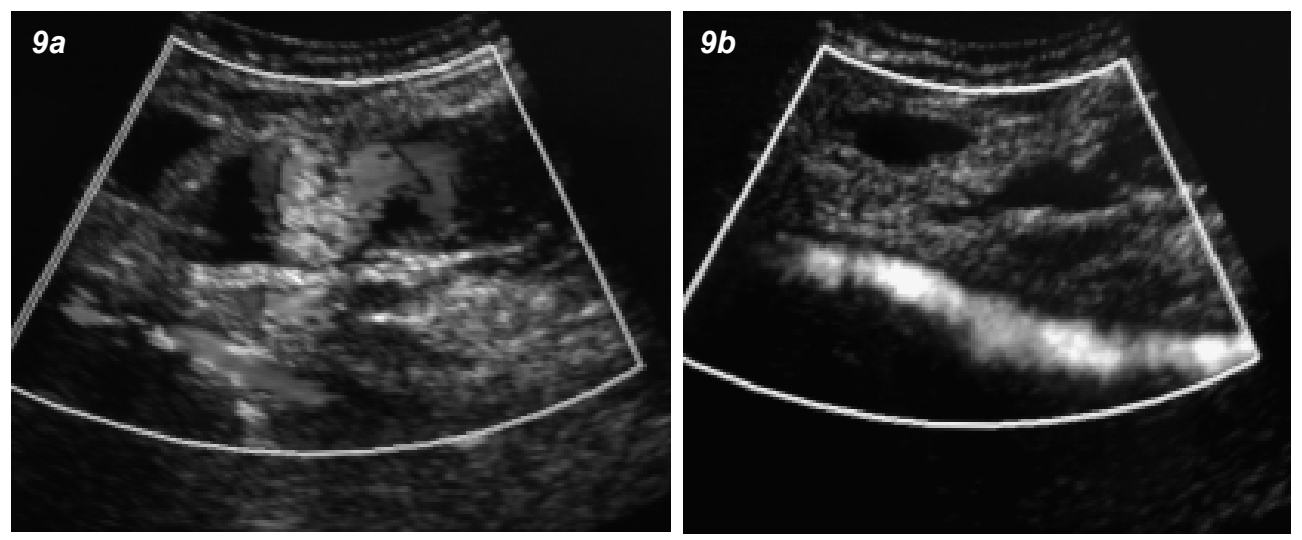

Figura 9 a,b. Tratamiento compresivo de pseudoaneurisma femoral post- coronariografía monitorizado con US Doppler color. a: Control previo a la compresión. $b$ : US Post-compresión demostrando un adecuado control del pseudoaneurisma.

drenajes de abscesos, ablación de tumores o accesos venosos. El éxito de estos procedimientos dependerá de una adecuada evaluación y control del paciente antes, durante y posterior al procedimiento efectuado.

\section{Bibliografía}

1. Reading CC, Charboneau JW, James EM, Hurt MR. Sonographically guided percutaneous biopsy of small ( $3 \mathrm{~cm}$ or less) masses. AJR Am J Roentgenol. 1988; 151: 189-192.

2. Torriani M, Etchebehere M, Amstalden E. Sonographically guided core needle biopsy of bone and soft tissue tumors. J Ultrasound Med 2002; 21: 275-281.

3. Smith EH. Complications of percutaneous abdominal fine-needle biopsy. Review. Radiology 1991; 178: 253258.

4. Kim SH, Lim HK, Lee WJ, Cho TM, Jang HJ. Needletract implantation in hepatocellular carcinoma: frequency and CT findings after biopsy with a 19.5gauge automed biopsy gun. Abdom Imaging 2000; 25: 246-250.

5. Stangl R, Altendorf-Hofmann A, Charnley RM, Scheele $J$. Factors influencing the natural history of the colorectal liver metastases. Lancet 1994; 343: 140510.

6. Rossi S, Stasi MD, Buscarini E, Garbangnani F, et al. Percutaneus treatment of small hepatic tumors by an expandable RF needle electrode. AJR Am J Roentgenol 1998; 170:1015-1022.

7. Venkataramu NK Krishnamurthy V, Casillas J; Latorre L. Radio-frequency ablation of hepatic lesions: A review. Appl Radiol 2003; 32: 11-26.

8. Solbiati L, lerace T, Golberg SN, et al. Percutaneous US-guided radio-frequency tissue ablation of liver metastases: treatment and follow-up in 16 patients. Radiology 1997; 202: 195-203.

9. Livraghi T, Goldberg N, Lazzaroni S. et al. Small hepatocelular carcinoma: treatment with radiofrecuency ablation versus ethanol injection. Radiology 1999; 210: 655-661.

10. Dongil Choi, Hyo K.Lim, Min Ju Kim et al. Recurrent Hepatocellular carcinoma: Percutaneous Radiofrequency ablation after hepatectomy. Radiology 2004; 230: 135-141.

11. Rhim H, Goldberg SN, Dodd GD. III et al. Essential techniques for successful radio-frequency thermal ablation of malignant hepatic tumors. Radiographics. 2001; 21: 17-35.

12. Lambiase RE, Deyoe L, Cronan JJ, Dorman GS. Percutaneous drainage of 335 consecutive abscesses: results of primary drainage with a 1 year follow-up. Radiology 1992; 184: 167-179.

13. Giorgio A, Tarantino L, Mariniello N, et al. Pyogenic liver abscesses: 13 years of experience in percutaneous needle aspiration with us guidance. Radiology. 1995; 195: 122-124.

14. Steiner E, Mueller PR, Hahn PF, et al. Complicated pancreatic abscesses: problems in interventional management .Radiology 1988; 167: 443-446.

15. vanSonnenberg E, Wing VW, Casola $G$ et al. Temporizing effect of percutaneous drainage of complicated abscesses in critically ill patients. AJR Am J Roentgenol 1984; 142: 821-826.

16. Duszak RL Jr, Levy JM, Akins EW, et al Percutaneous catheter drainage of infected intra-abdominal fluid collections. American College of Radiology. ACR Appropriateness Criteria Radiology 2000; 215 (suppl): 1067-1075.

17. David M. Hovsepian DM. Transrectal and transvaginal abscess drainage. J Vasc Interv Radiol 1997; 8: 501515.

18. Harrisinghani MG, MD, Gervais DA, MD, Maher MM et al. Transgluteal approach for percutaneous drainage of deep pelvic abscesses: 154 cases. Radiology 2003; 228: 701-705.

19. Vingan HL, Wohlgemuth SD, Bell JS 3d. Percutaneous cholecystostomy drainage for the treatment of acute emphysematous cholecystitis . AJR Am J Roentgenol 1990; 155: 1013-1014.

20. Lambiase RE, Cronan JJ, Dorfman GS et al. Tubeless percutaneous renal surgery: review of first 112 patients. Urology 2002; 59: 527-531.

21. Gray RR, So CB, McLoughlin RF et al. Outpatient percutaneous nephrostomy. Radiology 1996; 198: 8588.

22. Ramchandani P, Cardella JF, Grassi CJ et al. Quality improvement guidelines for percutaneous nephrostomy. J Vasc Interv Radiol 2001; 12: 12471251.

23. Gordon AC, Saliken JC, Johns D et al. US guided puncture of the internal jugular vein: complications and anatomic considerations. J Vasc Interv Radiol 1998; 9: 333-338. 